\title{
Research Paper \\ Effect of Acupressure at ShenMen Acupoint on the Sleep Quality of Nurses in Emergency Departments and Intensive Care Units
}

\author{
Sajjad Abbasi Tadi ${ }^{1}$ (1), ${ }^{*}$ Mahdieh Saberi ${ }^{1}$ (1), Hossein Akbari²
}

1. Department of Nursing, School of Nursing and Midwifery, Kashan University of Medical Sciences, Kashan, Iran.

2. Department of Biostatistics, School of Health, Kashan University of Medical Sciences, Kashan, Iran.

\begin{tabular}{|c|c|}
\hline $\begin{array}{l}\text { Use your device to scan } \\
\text { and read the article online }\end{array}$ & \\
\hline ajpirita & $\begin{array}{l}\text { Citation: Abbasi Tadi S, Saberi M, Akbari H. [Effect of Acupressure at ShenMen Acupoint on the Sleep Quality of Nurses in } \\
\text { Emergency Departments and Intensive Care Units (Persian)]. Complementary Medicine Journal. 2021; 10(4):380-395. https:// } \\
\text { doi.org/10.32598/cmja.10.4.1025.1 }\end{array}$ \\
\hline 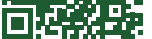 & dol'https://doi.org/10.32598/cmja.10.4.1025.1 \\
\hline
\end{tabular}

\section{(i) (3)}

Article Info:

Received: 13 Oct 2020

Accepted: 01 Dec 2020

Available Online: 01 Jan 2021

Key words:

Acupressure, Point (HT7), Sleep quality, Nurses

\section{ABSTRACT}

Objective Sleep disorders in nurses have a significant impact on their patient care quality. This study aims to evaluate the effect of acupressure at ShenMen acupoint (HT7) on the sleep quality of nurses in emergency departments and Intensive Care Units (ICUs).

Methods In this randomized clinical trial with pre-test-post-test design with control group, participants were 80 nurses working in emergency departments and ICUs of hospitals in Kashan, Iran. There were divided into two groups of intervention and control using the block randomization method after signing a written informed consent form. The intervention group underwent acupressure intervention at HT7 in both hands twice per day for 4 weeks. The control group did not receive any intervention. Data collection tools were a demographic form and Pittsburgh Sleep Quality Index (PSQI) questionnaire. Collected data were analyzed in SPSS software using descriptive statistics and independent t-test, Mann-Whitney U test, Chi-square test, and paired t-test, considering a significance level of 0.05 .

Results Pre-test results showed no statistically significant difference in the Mean \pm SD score of PSQI between the two groups of intervention $(6.94 \pm 1.96)$ and control $(7.53 \pm 3.57)(P>0.05)$, but the post-test results showed a significant increase in the mean score of PSQI in the intervention group (4.48 \pm 1.61 ) compared to the control group $(6.83 \pm 2.81)(P<0.001)$.

Conclusion Acupressure on ShenMen acupoint can increase the sleep quality of nurses; therefore, it is recommended for the management of their sleeps.

\section{Extended Abstract}

\section{Introduction}

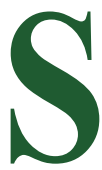

leep as an important and influential factor in health status, plays an important role in improving the quality of life such that its impairment can have severe negative effects on physical and mental health and, thus, fatigue and drowsiness during the day, which in turn reduces a person's daily functioning. Nurses are exposed to relatively high stress when providing care to the patient, due to high patient care responsibility and factors associated with their sleep disorders such as long hours of working that put them under emotional and psychological pressure. The effect of patient care necessity can increase their stress, fatigue, and sleep disturbance and may lead to errors that can adversely affect the patient's recovery or lead to adverse consequences such as their death. The results of some studies have indicatincrease healthcare costs. Sleep disorders lead to increased

\section{* Corresponding Author:}

Mahdieh Saberi, PhD.

Address: Department of Nursing, School of Nursing and Midwifery, Kashan University of Medical Sciences, Kashan, Iran.

Tel: +98 (913) 2606413

E-mail: dsabery@yahoo.com 
ed an unsatisfactory level of sleep quality among nurses. For example, Bozorg Sohrabi et al. reported a low level of sleep quality in $84 \%$ of nurses [6]. In recent years, the interest of people in using complementary medicine has increased due to its positive effects on the some disorders. Acupressure is a form of complementary medicine with an easy technique that has received a lot of attention.

\section{Materials and Methods}

The present study is a controlled randomized clinical trial with a pre-test-post-test design. The study population consisted of all nurses working in the emergency departments and Intensive Care Units (ICUs). Samples were divided into two groups of intervention $(n=40)$ and control $(n=40)$ using the block randomization method. A demographic form and Pittsburgh Sleep Quality Index (PSQI) questionnaire were used for collecting data before and after the intervention. First, for 15 minutes, face-to-face training on how to apply acupressure, finding the desired points and applying pressure on them was presented to the nurses by a researcher who had a certificate in acupressure. The nurses were asked to perform the acupressure intervention once in the presence of the researcher to ensure their learning. They were then asked to perform the intervention twice a day for 4 weeks in both hands at short intervals using a pressure of 3-5 kg; once during work shift breaks or rest at home or anywhere else, and once at night 30 minutes before going to bed or in bed for 5 minutes. Nurses in the control group did not receive any intervention.

\section{Results}

The Mean \pm SD age of nurses in the intervention and control groups were $28.55 \pm 4.01$ and $28.68 \pm 3.40$ years, respectively, but there was no statistically significant difference between the two groups in terms of age $(\mathrm{P}=0.58)$. The mean of shift work per month of nurses in the intervention and control groups were $26.7 \pm 2.12$ and $26.92 \pm 2.41$, respectively. There was no statistically significant difference between the two groups in any demographic characteristics which indicates the homogeneity of samples in the two groups (Table 1). Within-group comparison using independent ttest in the intervention group showed a statistically signifi-

Table 1. Demographic characteristics of nurses in two study groups

\begin{tabular}{|c|c|c|c|c|}
\hline \multirow{2}{*}{\multicolumn{2}{|c|}{ Variables }} & \multicolumn{2}{|c|}{ No. (\%) } & \multirow{2}{*}{ Sig. } \\
\hline & & Intervention Group & Control Group & \\
\hline \multirow{2}{*}{ Gender } & Male & $14(35)$ & $17(42.5)$ & \multirow{2}{*}{$0.49 *$} \\
\hline & Female & $26(65)$ & $23(57.5)$ & \\
\hline \multirow{2}{*}{ Marital status } & Single & $19(47.5)$ & $13(32.5)$ & \multirow{2}{*}{$0.17^{*}$} \\
\hline & Married & $21(52.5)$ & $27(62.5)$ & \\
\hline \multirow{4}{*}{ Number of children } & 0 & $28(70)$ & $21(17.5)$ & \multirow{4}{*}{$0.33^{* *}$} \\
\hline & 1 & $3(7.5)$ & $7(20)$ & \\
\hline & 2 & $7(17.5)$ & $8(10)$ & \\
\hline & 3 & $2(5)$ & 4 (19.4) & \\
\hline \multirow{2}{*}{ Educational level } & BA & $32(80)$ & 31 (77.5) & \multirow{2}{*}{$0.78 * *$} \\
\hline & $\mathrm{MSc} / \mathrm{MA}$ & $8(20)$ & $9(22.5)$ & \\
\hline \multirow{3}{*}{ Work experience } & $<5 y$ & $20(50)$ & $21(52.5)$ & \multirow{3}{*}{$0.82^{* *}$} \\
\hline & $5-10 y$ & $13(32.5)$ & $14(35)$ & \\
\hline & $>10 y$ & $7(17.5)$ & $5(12.5)$ & \\
\hline \multirow{4}{*}{ Number of night shifts } & 0 & $6(15)$ & $10(25)$ & \multirow{4}{*}{$0.38^{* *}$} \\
\hline & 1 & $14(35)$ & $9(22.5)$ & \\
\hline & 2 & $16(40)$ & $14(35)$ & \\
\hline & $>2$ & $4(10)$ & $7(17.5)$ & \\
\hline \multirow{2}{*}{ Shift schedule } & Fixed & $6(15)$ & $10(25)$ & \multirow{2}{*}{$0.26^{*}$} \\
\hline & Rotating & $34(85)$ & $30(75)$ & \\
\hline \multirow{2}{*}{ Department } & Emergency & $20(50)$ & $20(50)$ & \multirow{2}{*}{$1^{*}$} \\
\hline & $\mathrm{ICU}$ & $20(50)$ & $20(50)$ & \\
\hline
\end{tabular}


Table 2. Mean \pm SD of PSQI scores in two study groups before and after intervention

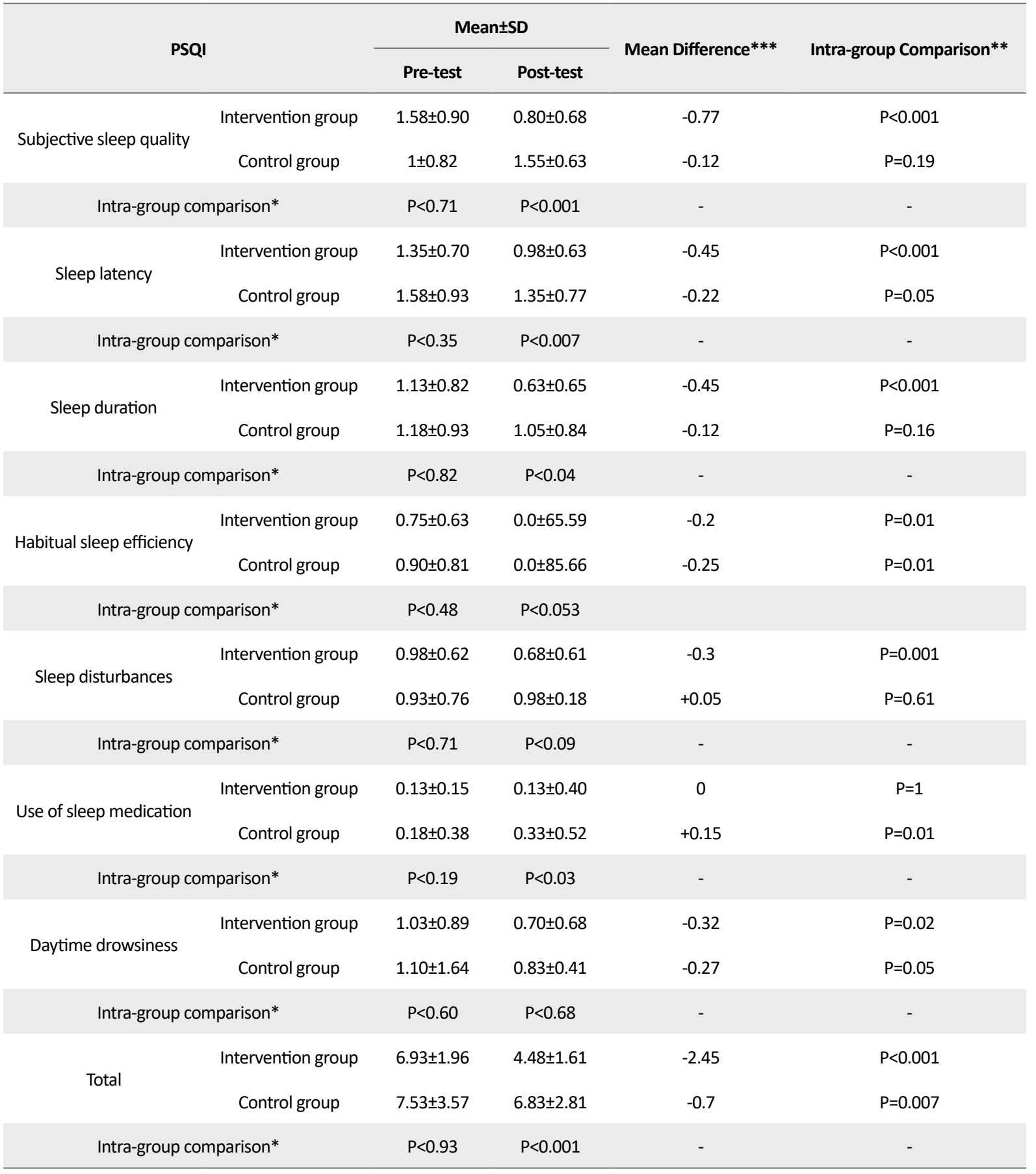

*Independent t-test; **Paired t-test; ***Repeated measures ANOVA.

cant decrease in PSQI dimensions of subjective sleep quality, sleep latency, sleep duration, habitual sleep efficiency, sleep disturbances, daytime drowsiness and in overall PSQI score after intervention $(\mathrm{P}<0.05)$. In the control group, the results of independent t-test showed a significant decrease in sleep latency, habitual sleep efficiency, use of sleep medication, daytime drowsiness and in overall PSQI score after the intervention $(\mathrm{P}<0.05)$. The results of paired $t$-test for be- tween-group comparison of sleep quality in the intervention group showed that the mean score of overall sleep quality increased but the scores of all subscales (except in use of sleep medication) decreased significantly after the intervention $(\mathrm{P}<0.05)$, while the mean score of overall PSQI and its subscales in the control group did not show a statistically significant difference compared to the scores of baseline $(\mathrm{P}<0.05)$ (Table 2). 


\section{Conclusion}

According to the results of the present study, the use of acupressure at Shenmen acupoint (HT7) for 4 weeks is effective in reducing sleep disorders in nurses working in emergency and ICUs. Therefore, due to the very high prevalence of insomnia and its complications in nurses, acupressure can be suggested as a non-pharmacological treatment in the management of nurses' sleep disorders and consequently the quality of life, due to ease of use and not having side effects.

\section{Ethical Considerations}

Compliance with ethical guidelines

This study was approved by Kashan University of Medical Sciences (Code: IR.KAUMS.NUHEPM. REC.1399.025) and has been registered by Iranian Registry of Clinical Trials.

\section{Funding}

This study was supported by the Deputy for Research of Kashan University of Medical Sciences.

Authors' contributions

Writing - original draft: Mahdieh Saberi; Writing - review \& editing, data analysis: Hossein Akbari; Data collection: Sajjad Abbasi Tadi.

\section{Conflicts of interest}

The authors declared no conflict of interest.

Acknowledgements

The authors would like to thank all nurses participated in the study, the Deputy for Research of Kashan University of Medical Sciences, and Director of Shahid Beheshti Hospital in Kashan for their cooperation. 
This Page Intentionally Left Blank 


\title{
بررسى تأثير طب فشارى نقطه شن من (HT7) بر كيفيت خواب يرستاران شاغل در بخش هایى اورخانس و مراقبتهائ ويزه (ICU)
}

\author{
سجاد عباسى طادى' ـ، "مهديه صابرى' ه، حسين اكبرى' \\ 1. كروه هيرستارى، دانشكده هيرستارى و مامايى، دانشعاه علوميزشكى كاشان، كاشان، ايران.

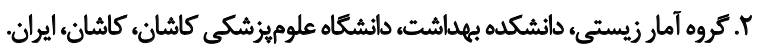

\begin{abstract}
يكيد

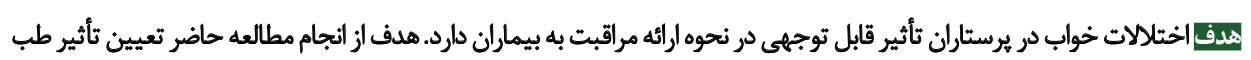

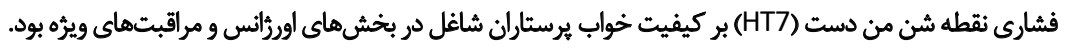

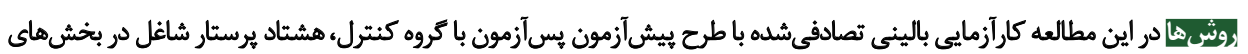

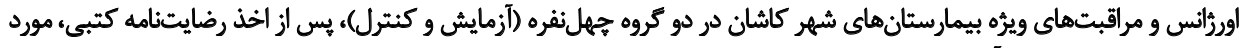

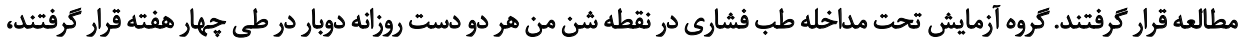

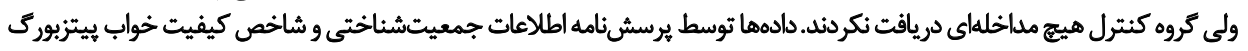

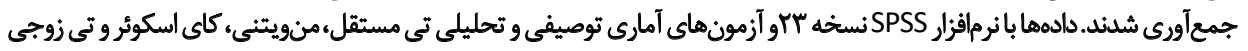

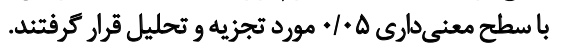

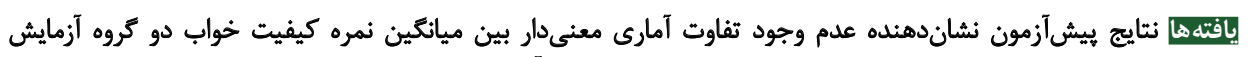

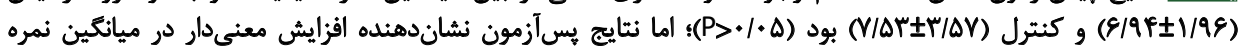

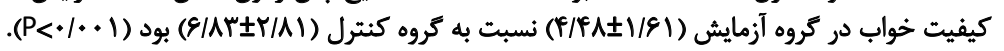

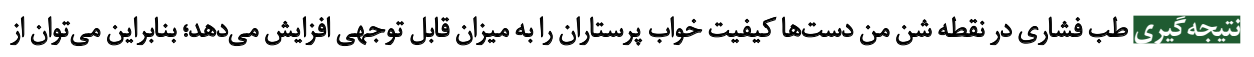

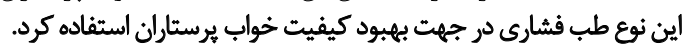

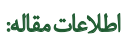

تاريخ دريافت: Tr مهر

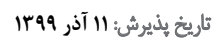

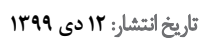

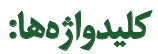
طب فشارى، نقطة شن

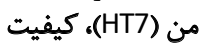
حوابه برستاران

ايجاد اختلالات خواب در آنان ارتباط مستقيم دارند [س]. در بين datio

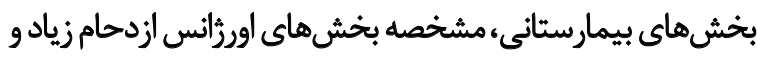

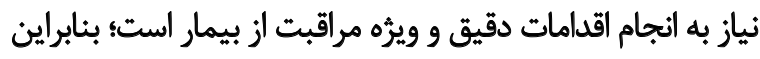

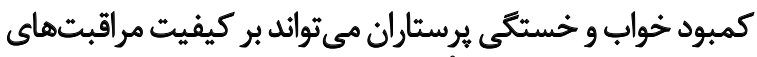

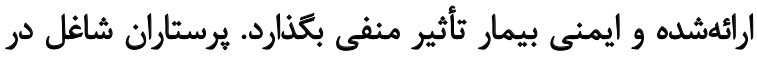

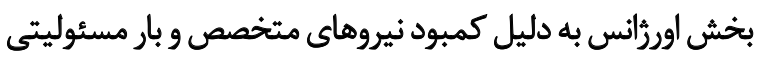

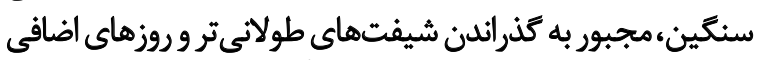

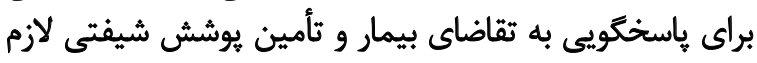

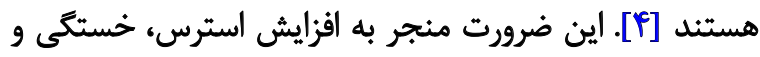

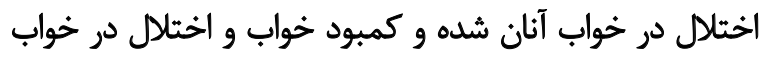

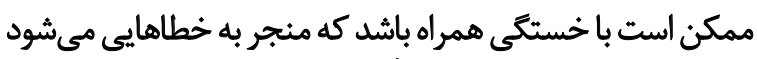

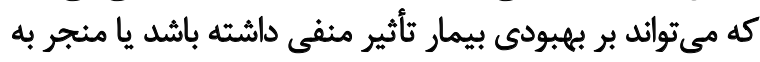

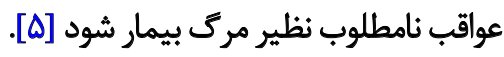
نتايج برخى مطالعات تأييدكنيده سطح نامطلوب كيفى

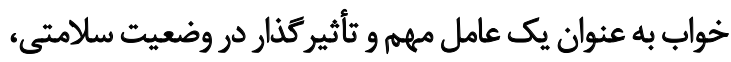

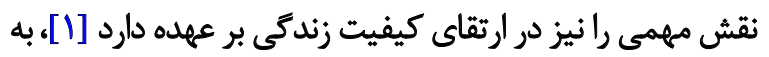

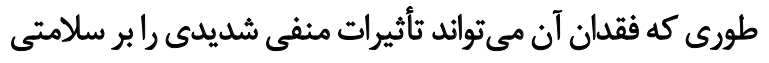

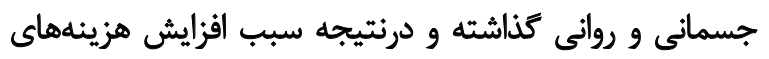

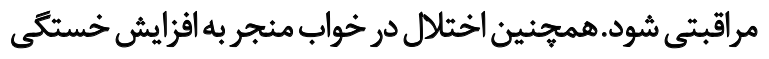

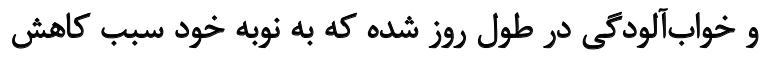

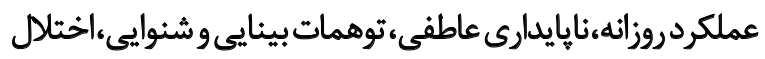

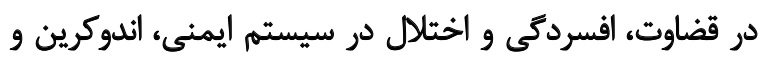

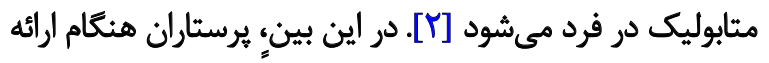

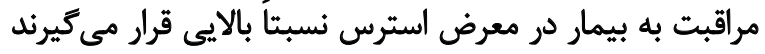

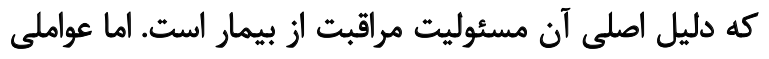

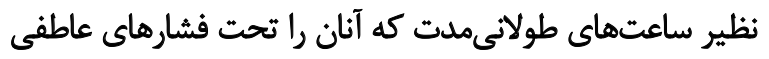

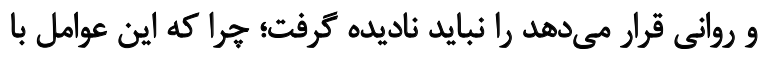

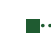




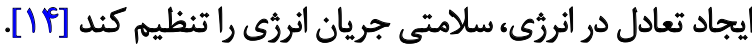

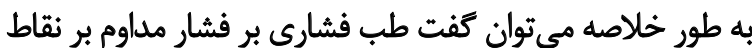

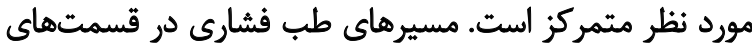

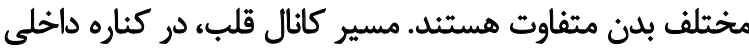

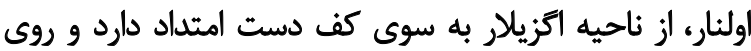

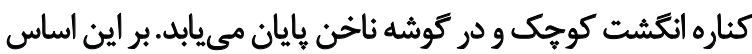

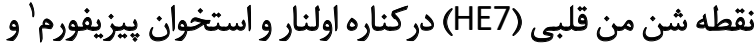

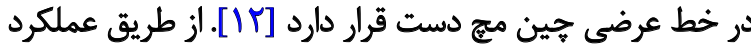

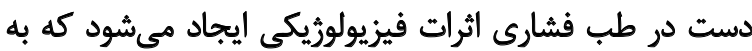

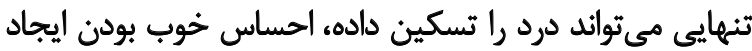

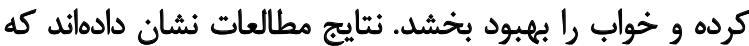

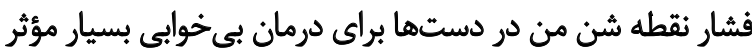

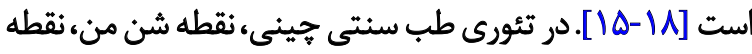

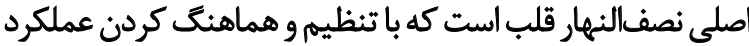

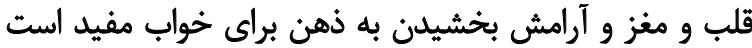

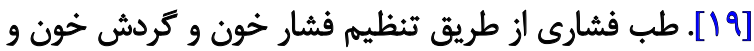

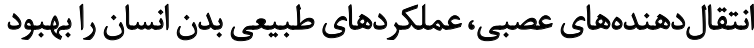

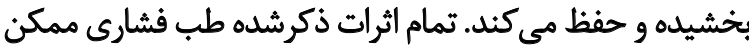

است كيفيت خواب را افزايش دهد [•r].

طب فشارى به علت كاربرد آسان، ارزان و غيرتهاجمى بوردن

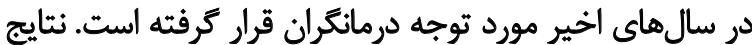

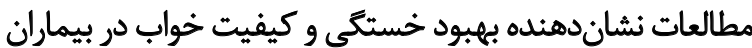

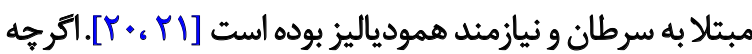

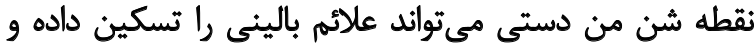

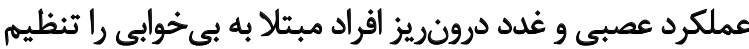

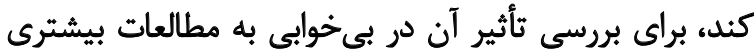

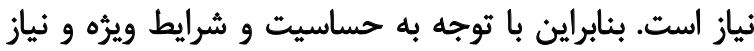

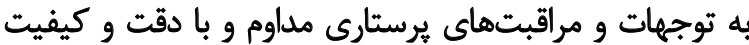

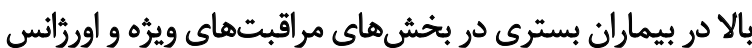

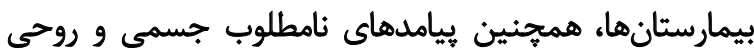

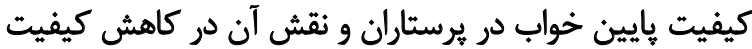

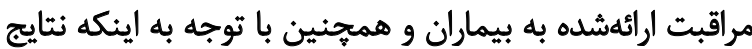

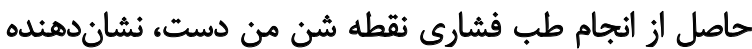

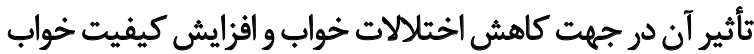

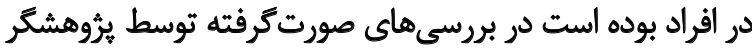

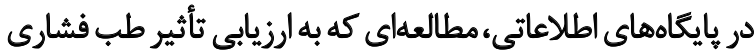

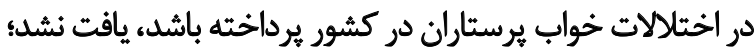

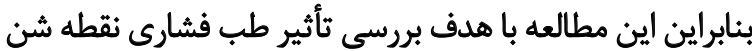

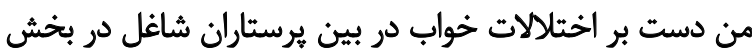
اوررانس و مراقبتهاى ويرٔه انجام شد.

1. Pisiform
خواب در بين يرستاران است؛ از جمله نتايج مطالعه سهرابي و

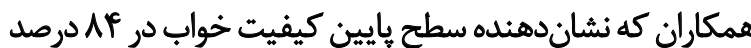

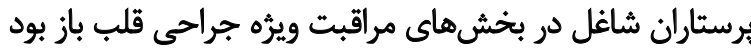

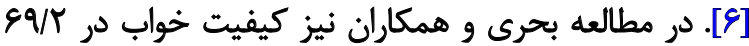

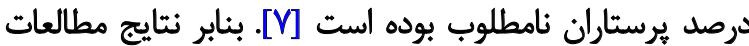

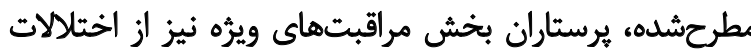

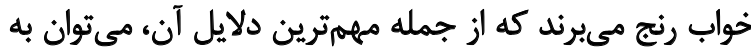

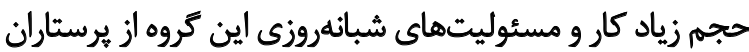

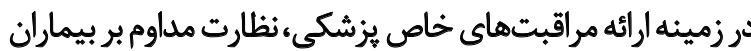

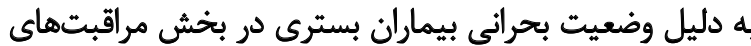

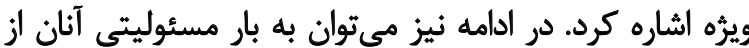

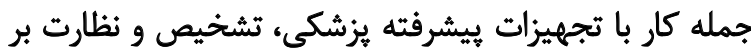

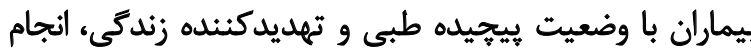

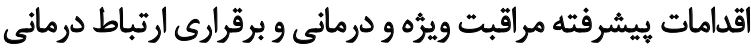
با بيماران و بستكان آنها نيز اشاره كرد [A].

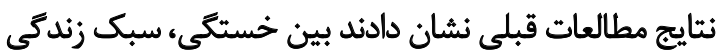

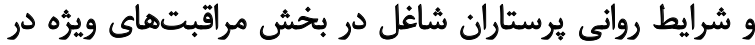

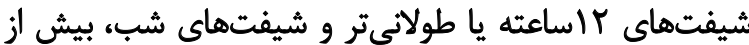

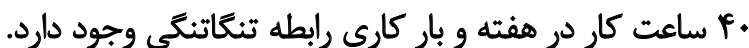

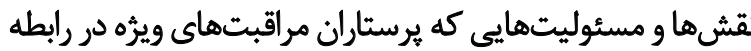

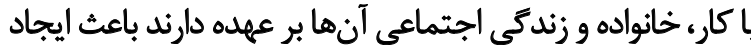

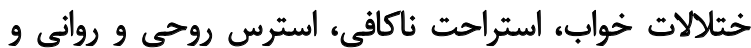

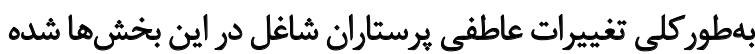

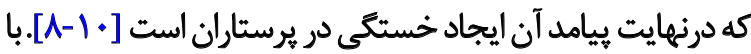

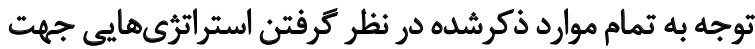

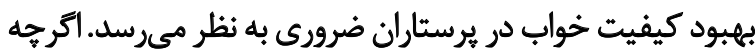

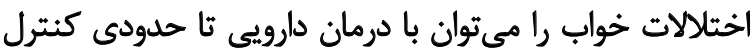

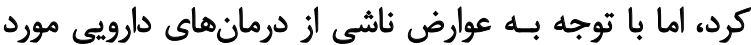

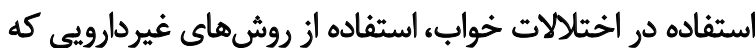

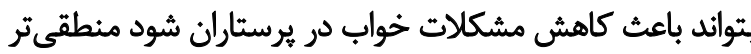

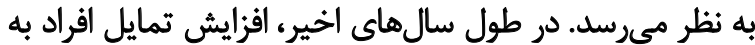

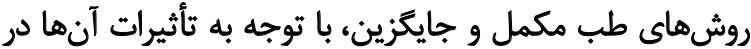

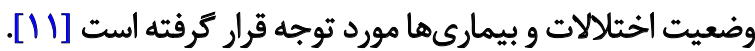

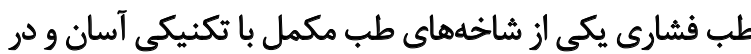

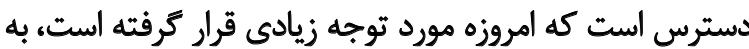

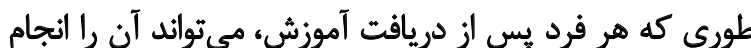

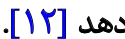

طب فشارى يكى از روشهاى طب سنتى جينى است و به به

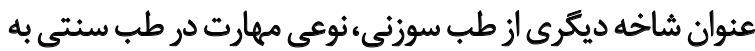

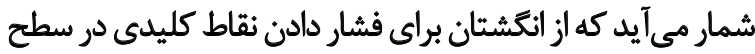

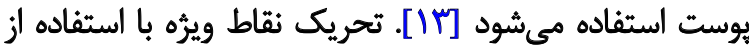

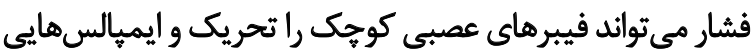

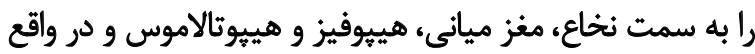
كانال هاى مخصوص در بدن انسان به نام نصفالنهار ارسال كند و بان ونيا 
فعاليت بالينى در بيمارستان، نوع شيفت، ميانكين تعداد شيفت

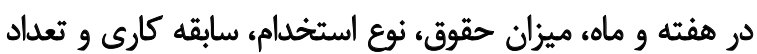

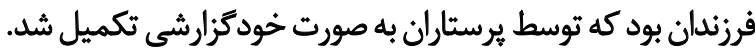

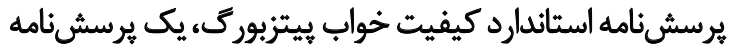

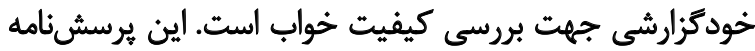

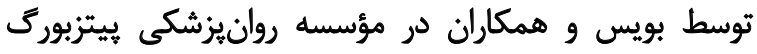

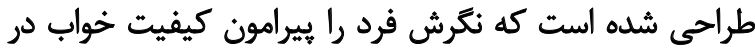

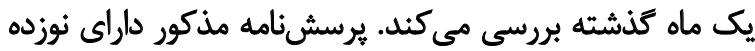

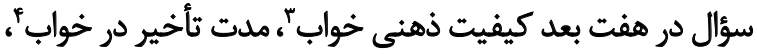

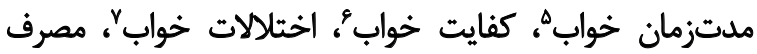

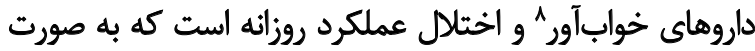

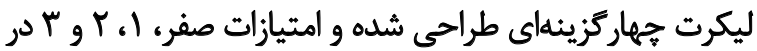

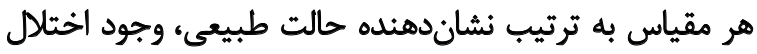

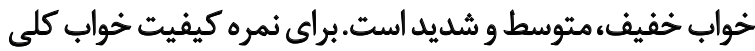

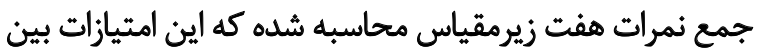

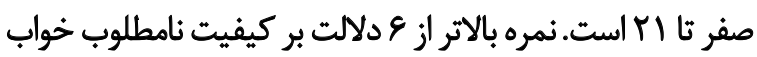

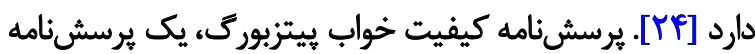

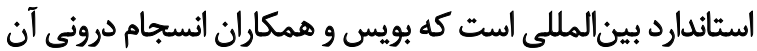

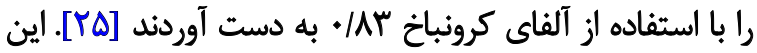

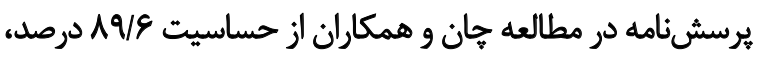

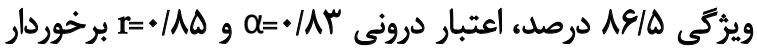

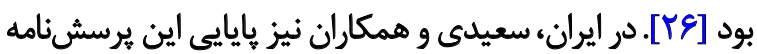

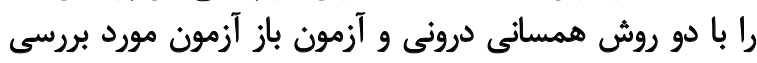

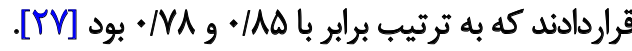

اين يُروهش يس از دريافت كد اخلاقيى از دانشعاه علوميزشكى

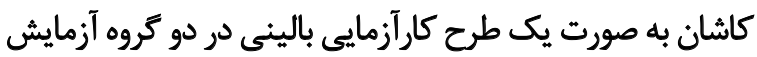

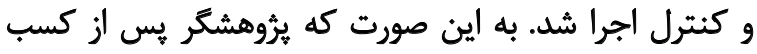

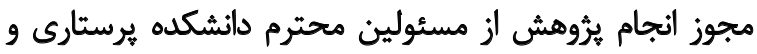

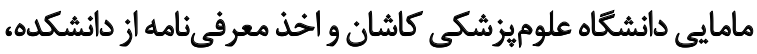

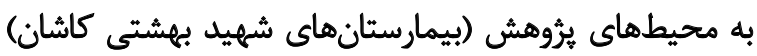

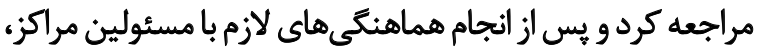

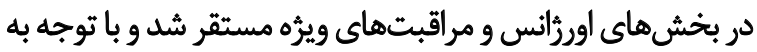

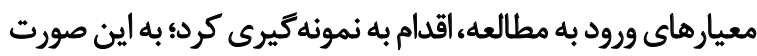

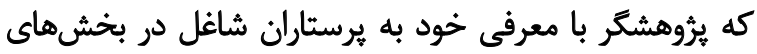

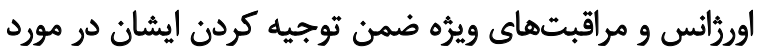

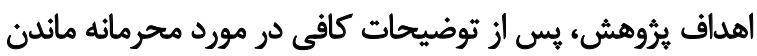

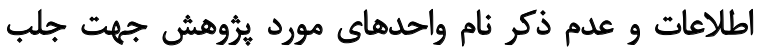
اعتماد آنها رضايتنامه كتبى از آنان اخذ كرد إندام

3. Subjective sleep quality

4. Sleep latency

5. Sleep duration

6. Habitual sleep efficiency

7. Sleep disturbances

8. Use of sleeping medication

\section{مواد و روشها}

ثيُوهش حاضر يك مطالعه كارآزمايى بالينى كنترلدار

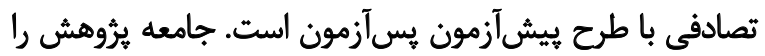

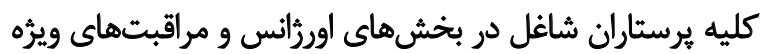

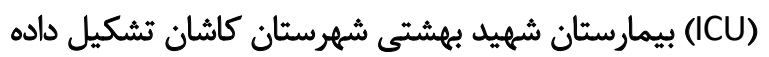

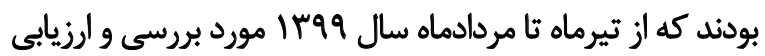

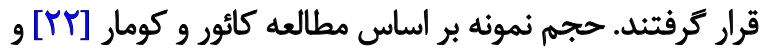

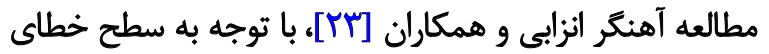

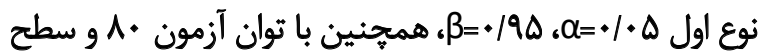

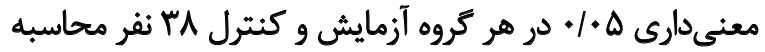

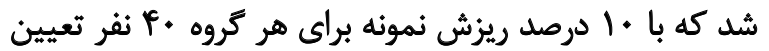

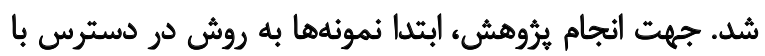

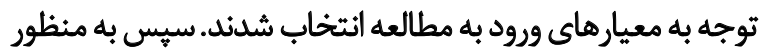

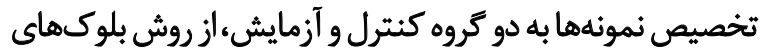

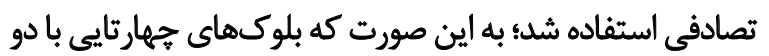

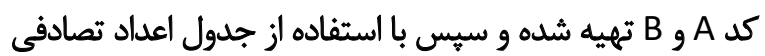

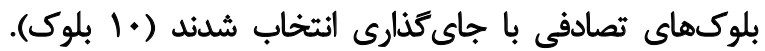

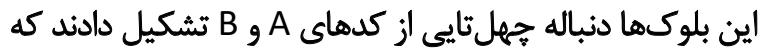

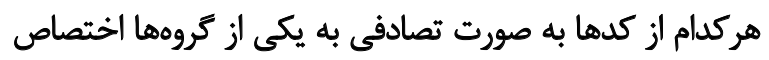

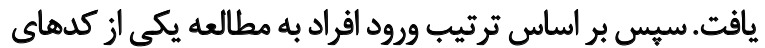
A (آزمايش) يا B (كنترل) را به خود اختصاص دادند.

معيارهاى ورود به مطالعه شامل دارا بودن حداقل دون دو سال

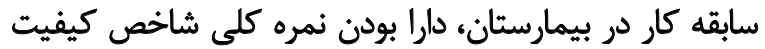

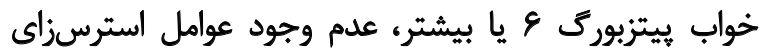

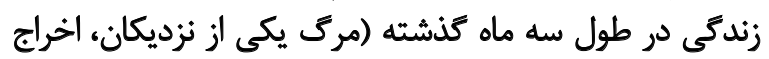

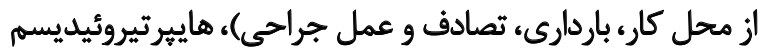

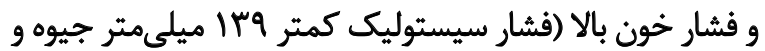

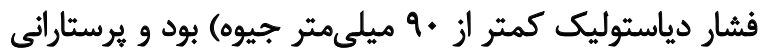

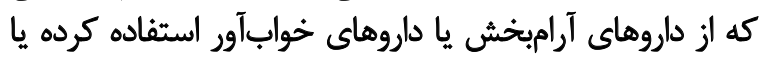

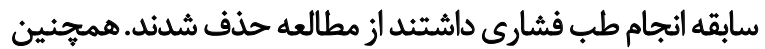

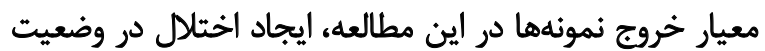

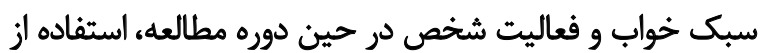

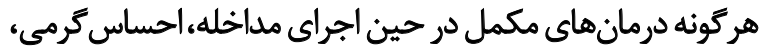

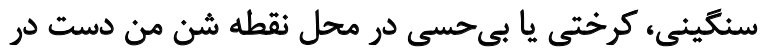

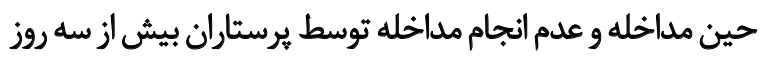
در طول هفته در نظر كرفته شد.

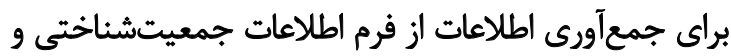

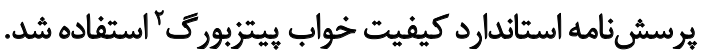

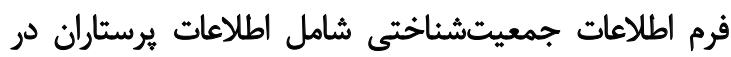

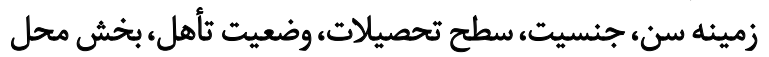




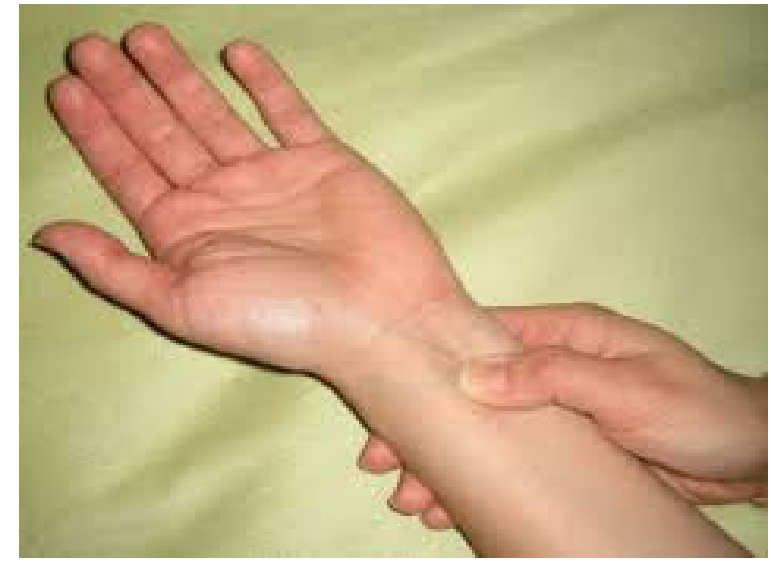

| تصوير ا. محل فشار نقطه شن من دست

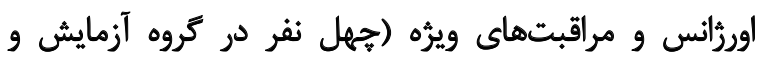

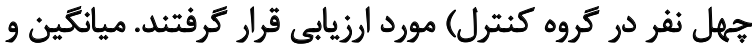

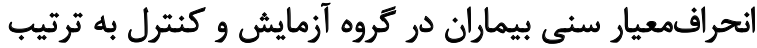

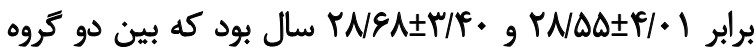

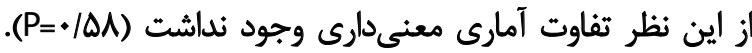

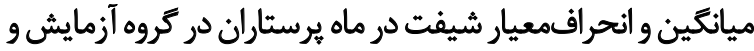
كنترل به ترتيب r

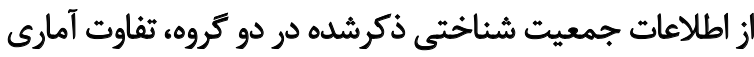

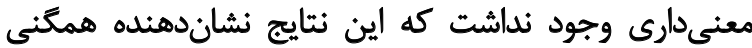
واحدهاى يثوهش در دو تروه است (جدول شماره ل).

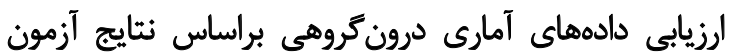

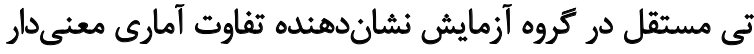

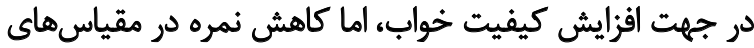

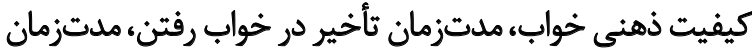

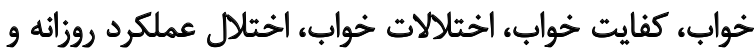

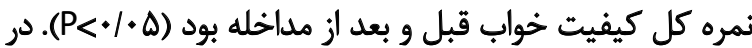

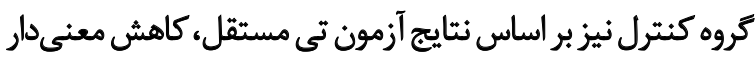

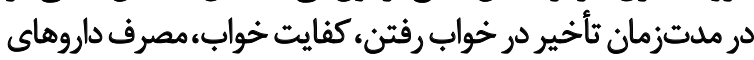

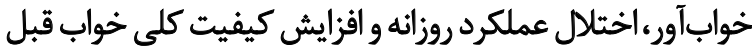

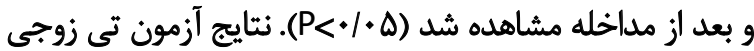

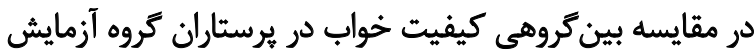

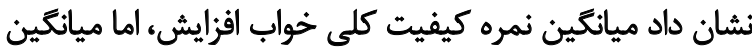

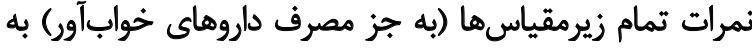

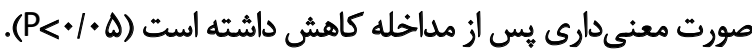

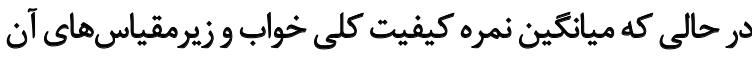

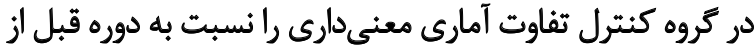

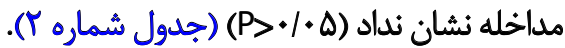

بحث

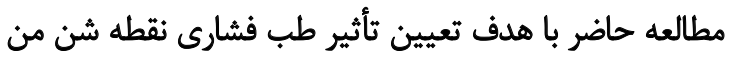

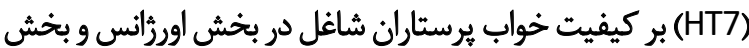

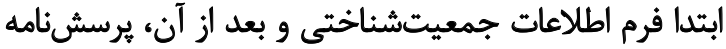

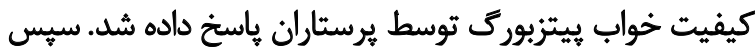

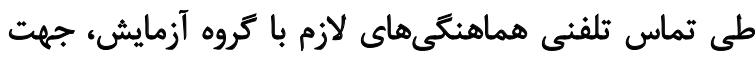

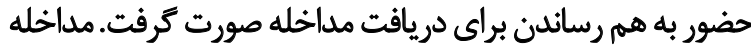

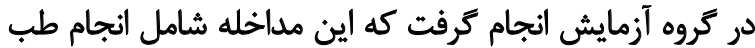

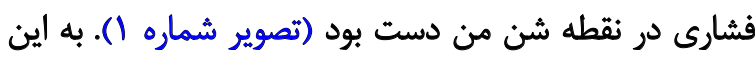

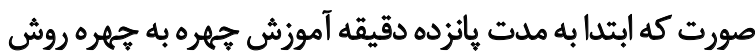

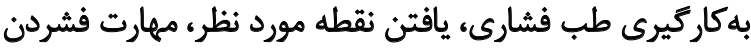

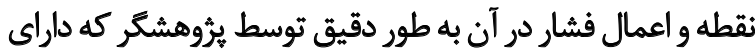

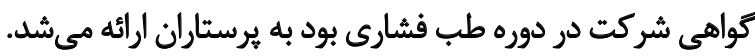

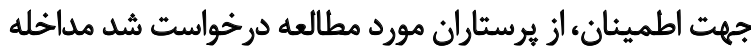

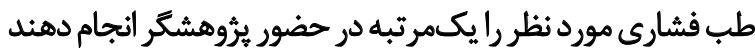

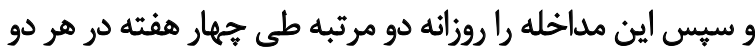

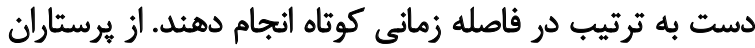

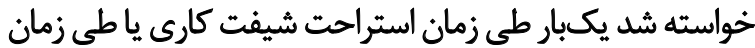

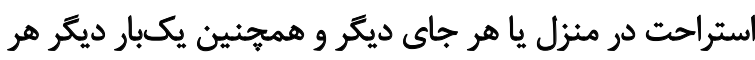

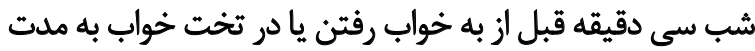

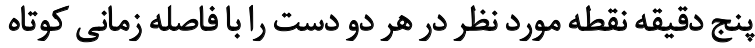

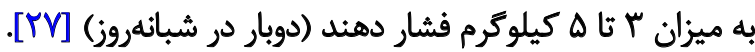

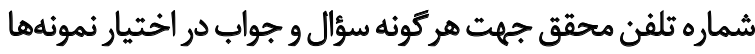

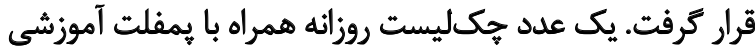

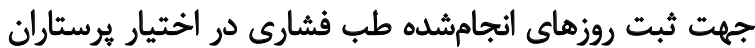

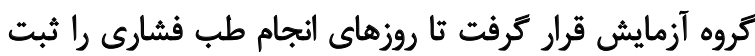

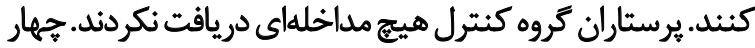

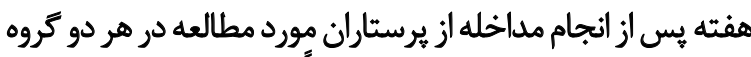

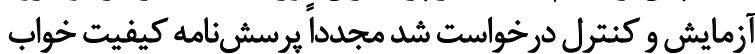

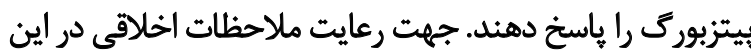

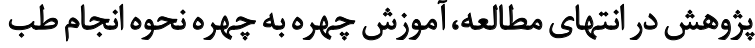

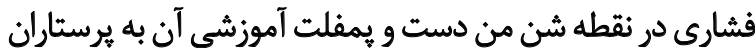
كروه كنترل داده شد.

تجزيه و تحليل دادهها با استفاده از نرمافززار SPSS نسخه تئن

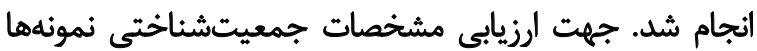

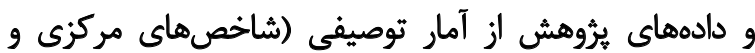

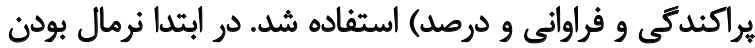

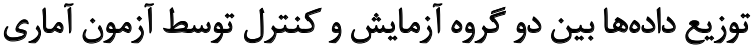

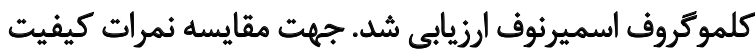

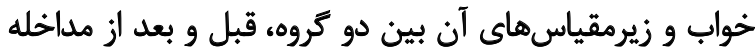

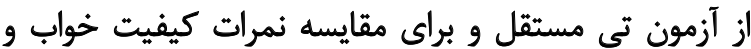

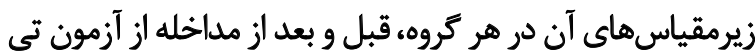

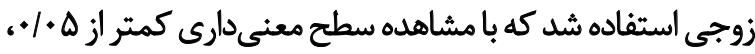

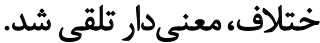

يافتهها

در اين مطالعه هشتاد نفر از برستاران شاغل در بخشهاى 
جدول ا. مقايسه خصوصيات جمعيتشناختى در دو گروه آزمايش و كنترل

\begin{tabular}{|c|c|c|c|c|}
\hline \multirow{2}{*}{ سطح معنى دارى } & \multicolumn{2}{|c|}{ تعداد (درصد) } & \multirow{2}{*}{\multicolumn{2}{|c|}{ متغير مورد بررسى }} \\
\hline & كروه كتترل & تروه آزمايش & & \\
\hline \multirow{2}{*}{.$/ 49^{\circ}$} & $\operatorname{IV}(\varphi T / \Delta)$ & $\| f(r \omega / \cdot)$ & مرد & \multirow{2}{*}{ جنسيت } \\
\hline & $\pi(\Delta V / \Delta)$ & $r E(\& D / \cdot)$ & ن & \\
\hline \multirow{2}{*}{.$/ 1 v^{*}$} & Ir/ & $19(F V / \Delta)$ & مجرد & \multirow{2}{*}{ وضعيت تأهل } \\
\hline & $\operatorname{Tr}(q Y / \Delta)$ & $M(\Delta T / \Delta)$ & متأهل & \\
\hline \multirow{4}{*}{ 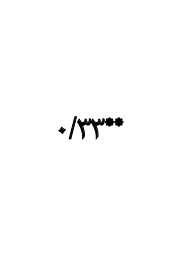 } & $M(I V / \Delta)$ & $M \Lambda(V+1 \cdot)$ & بدون فرزند & \multirow{4}{*}{ تعلداد فرزئدان } \\
\hline & $r(r .1 *)$ & $r(V / \Delta)$ & يك فرزند & \\
\hline & $A(1 . \%)$ & $V(I V / \Delta)$ & دو فرزئد & \\
\hline & $P(19 / 4)$ & $r(\Delta / \cdot)$ & سه فرزند & \\
\hline \multirow{2}{*}{$\cdot / \mathrm{NA}$} & $M(W / \Delta)$ & $M(A \cdot / \cdot)$ & كارشناسى & \multirow{2}{*}{ سطح تحصيلات } \\
\hline & $9(K / \Delta)$ & $\Lambda(T+/)$ & كارششاسى ارشد & \\
\hline \multirow{3}{*}{./Ar" } & $M(\Delta Y / \Delta)$ & $r \cdot(\Delta \cdot / \cdot)$ & كمثر از هـ سال & \multirow{3}{*}{ سابقه كارى } \\
\hline & $\| f(r \Delta / \cdot)$ & $\mathbb{I r}(\Gamma / \Delta)$ & هـ الى •ا سال & \\
\hline & $\Delta(I r / \Delta)$ & $V(I V / \Delta)$ & بيشتر از +ا سال & \\
\hline \multirow{4}{*}{. } & $1 \cdot(\Gamma \Delta / \cdot)$ & $g(\mid \omega / \cdot)$ & بلدون شيفت & \multirow{4}{*}{ تعلداد شيفت شب } \\
\hline & $9(\pi r / \Delta)$ & If $(r \Delta / \cdot)$ & يك شيفت & \\
\hline & $\| f(r \Delta / \cdot)$ & $18(8 \% 1 \%)$ & دو شيفت & \\
\hline & $V(\mid V / \Delta)$ & $1 \cdot(\Gamma \Delta / \cdot)$ & بيشتر از دو شيفت & \\
\hline \multirow{2}{*}{. } & $1 \cdot(T \Delta / \cdot)$ & $g(\mid \omega / \cdot)$ & ثابت & \multirow{2}{*}{ نوع شيفت } \\
\hline & $r \cdot(V \mathrm{VQ} / \cdot)$ & $m p(A \Delta / \cdot)$ & در كردش & \\
\hline \multirow{2}{*}{.$\Lambda^{\bullet}$} & $r \cdot(\Delta+\%)$ & $r \cdot(\Delta \cdot / \cdot)$ & اورر|أس & \multirow{2}{*}{ بخش محل فعاليت } \\
\hline & $r \cdot(\Delta \cdot / \cdot)$ & $r \cdot(\Delta \cdot / \cdot)$ & مراقبتهاى ويره & \\
\hline
\end{tabular}

در اين ميان، نقطه شن من ميج دستها كه نقطه دروازه روح و راه

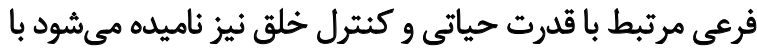

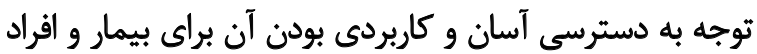

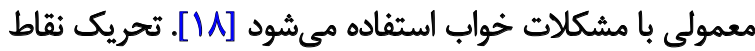

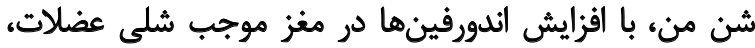

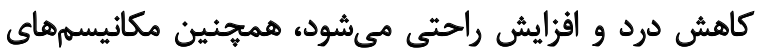

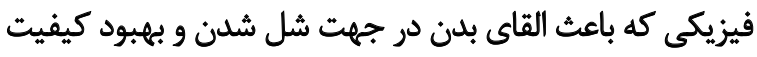

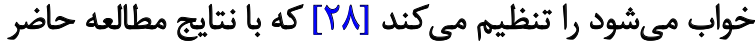

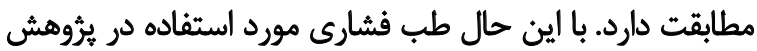

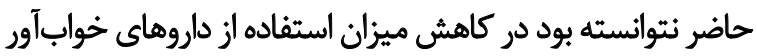

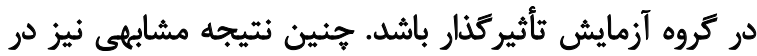

مراقبت ويرٔه انجام شد. بر اساس نتايج اين مطالعه، با توجه به ميه

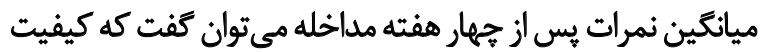

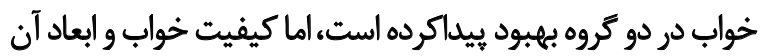

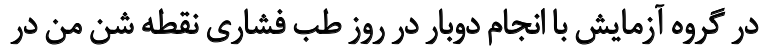

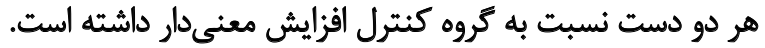

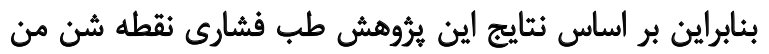

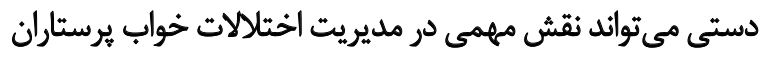
شاغل در بخش هاى اورثانس و مراقبتهائ ويره داشته باشد. بر اساس طب سنتى جينى نقاط زيادى هستند كه تحريك

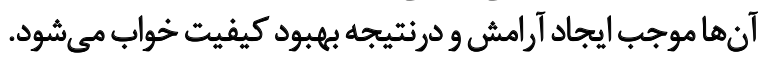


جدول ז. مقايسه ميانكين، انحرافمعيار و تغييرات نمرات زيرمقياسهاي شاخص كيفيت خواب دو كروه كثترل و آزمايش در دوره قبل از مداخله و جهار هفته يس از مداخله

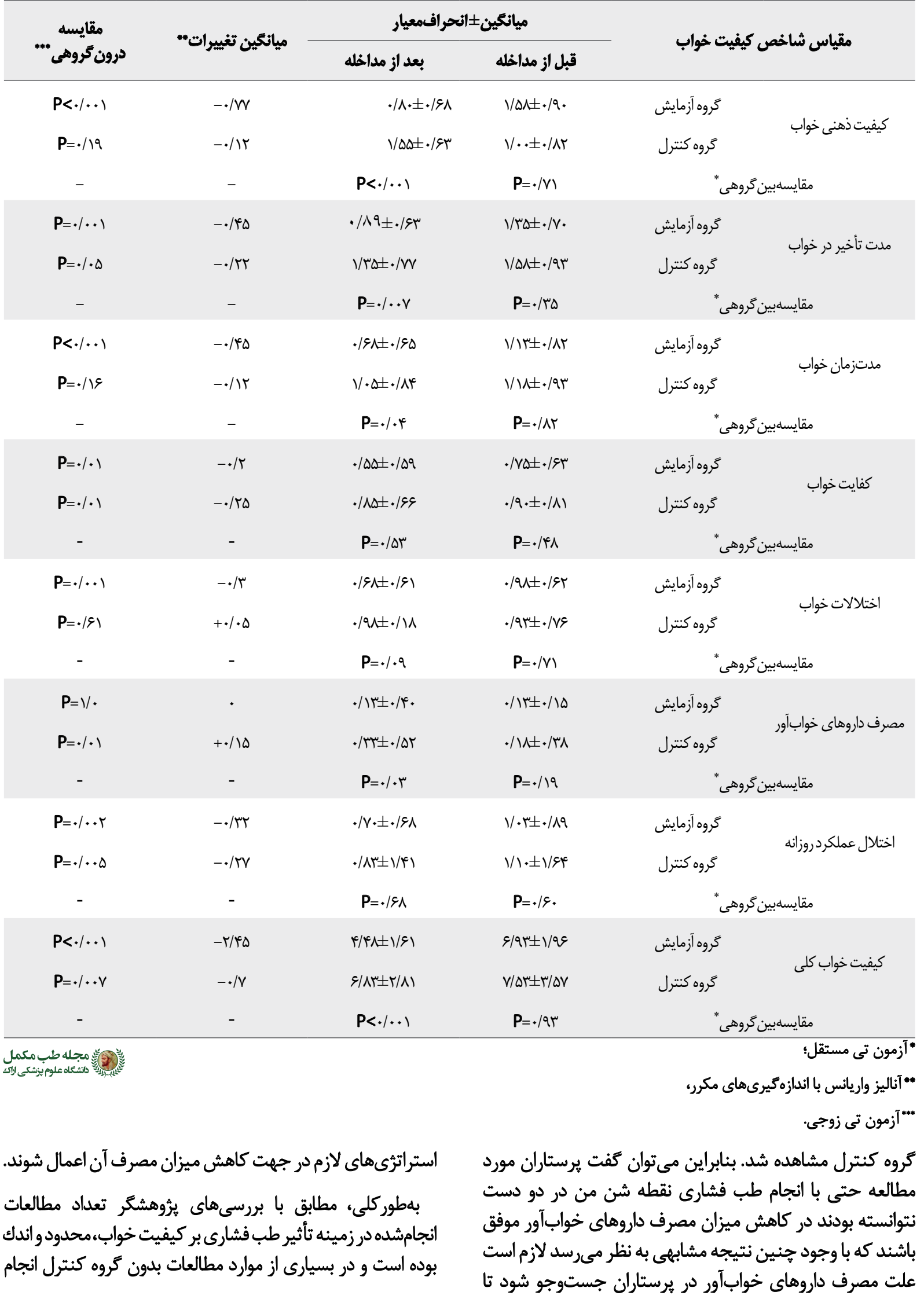




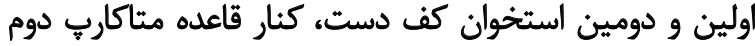

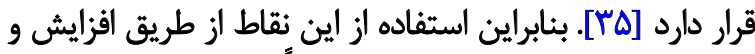

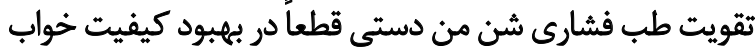

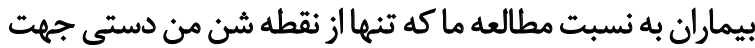

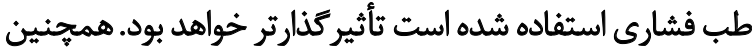

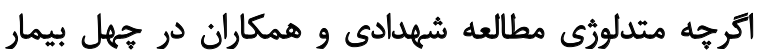
ديابتى تحت مداخله طب فشارى نقطه شن من دستى بـ با مطالعالعه

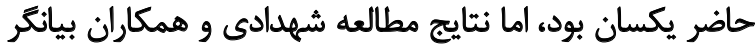

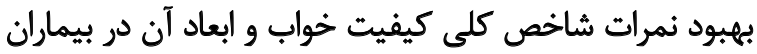

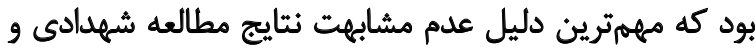

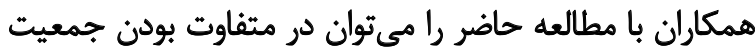

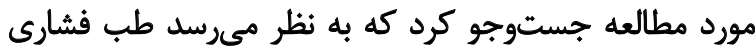

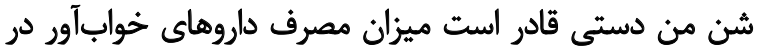

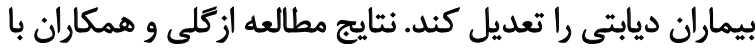

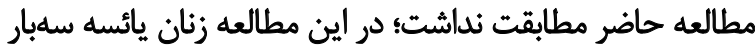

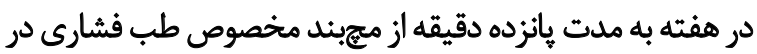

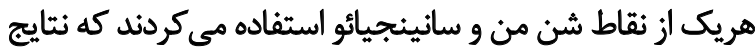

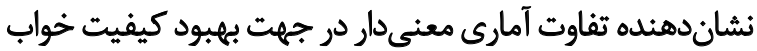

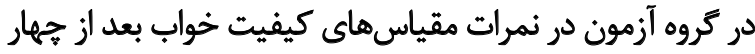

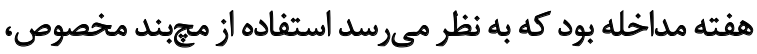

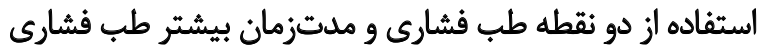

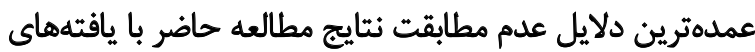

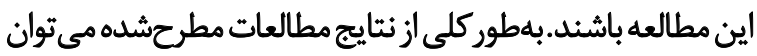

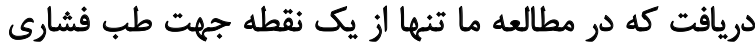

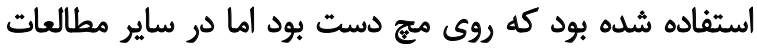

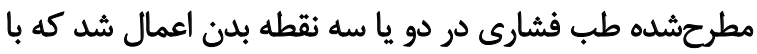

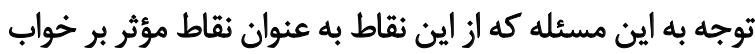

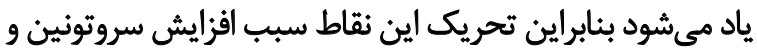

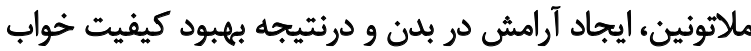
بيشتر در بين نمونههاي مورد مداخله مي ديودي.

با توجه به اين مسئله كه در تمامي مطالعات مطرحشده تفاوت

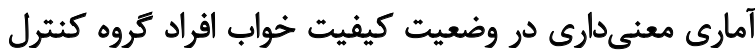

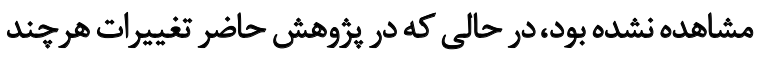

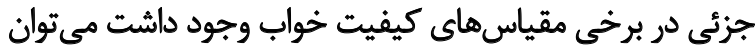

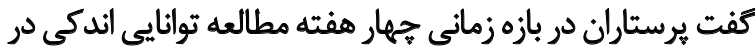

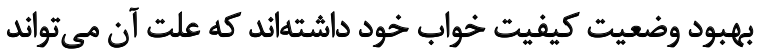

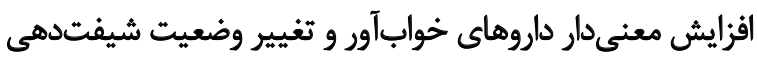

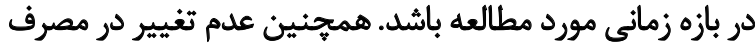

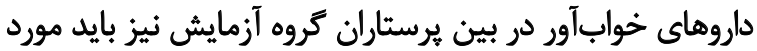

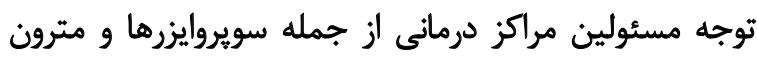

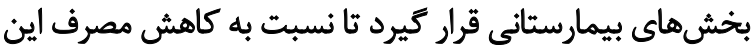

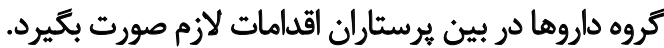

شده است. يافتههاى مطالعات سان و همكاران [ج [T]، سيمونسينى

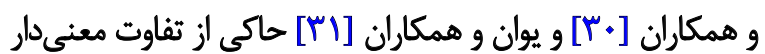

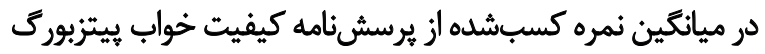

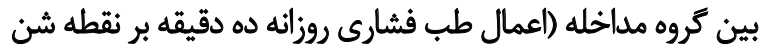

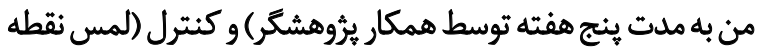

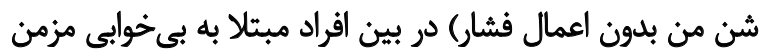

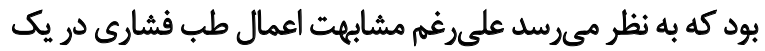

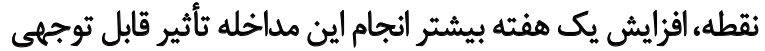
در كاهش ميزان مصرف داروهاى خواب داشته است. البته متفاوت بوني

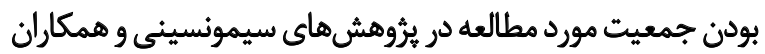
و يوان و همكاران را نبايد ناديده كرفت.

خ קندين مركز عصبى و انتقالدهندههاى عصبى در تنظيم

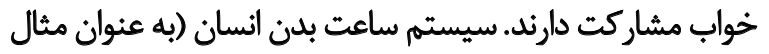

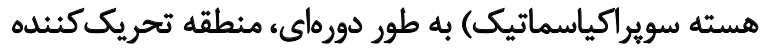

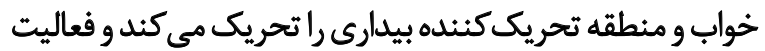

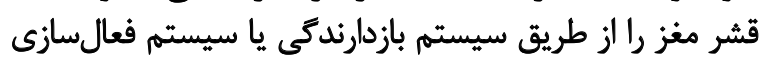

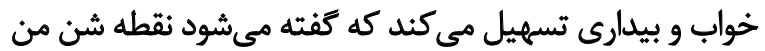

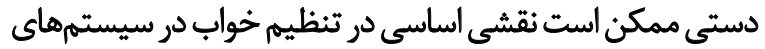

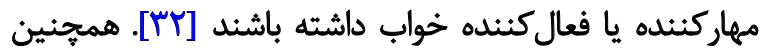

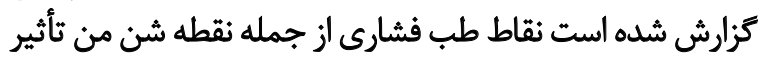

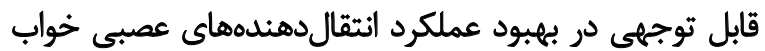

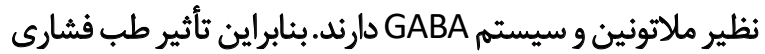

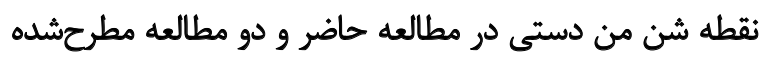

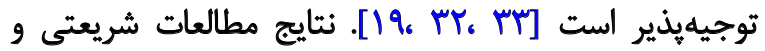

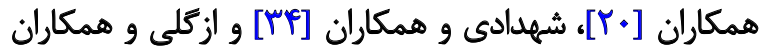

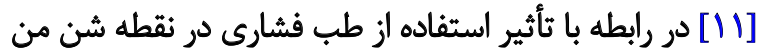

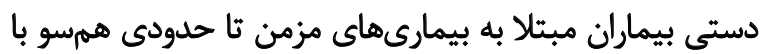

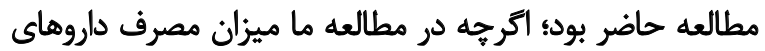

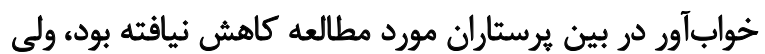

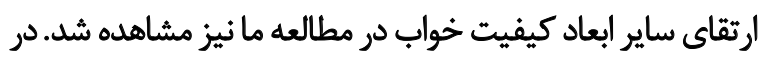

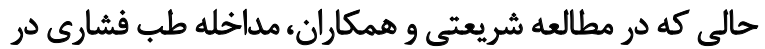

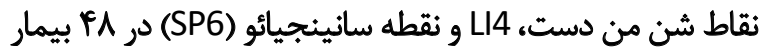

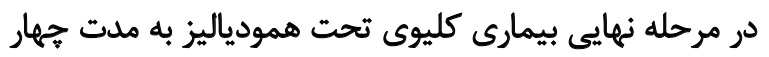

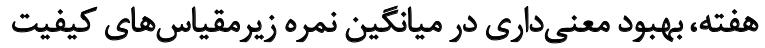
خواب و شاخص كلى خواب نشان داد. با توجه به به استفاده از سهانه

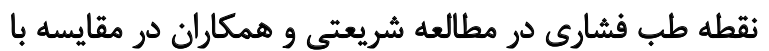

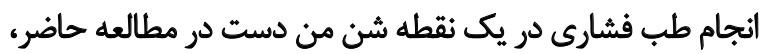

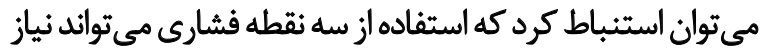

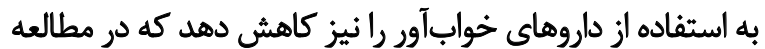

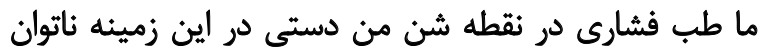

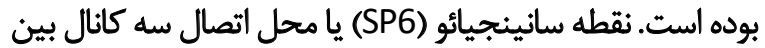

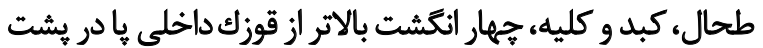

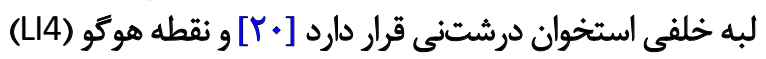

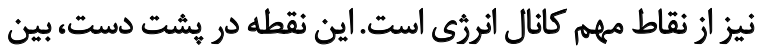




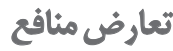

بنابر اظهار نويسندكان، اين مقاله تعارض منافع ندارد.

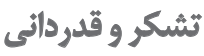

از كليه يرستاران شركت كنينده در ثرؤوهش، مديران و مسئولان

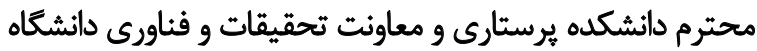

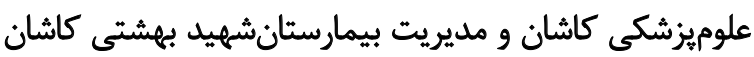
براى همكارى در اين مطالعه كمال تشكر و قدرداني را داريم.
با توجه به نتايج بهدستآمده از دادههاى آمارى در اين مطالعه،

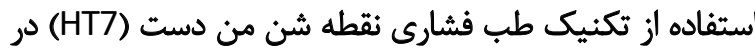

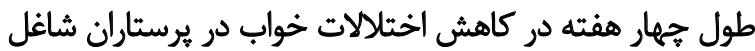

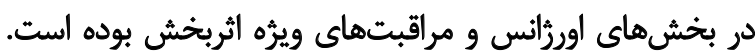

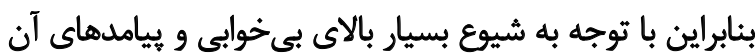

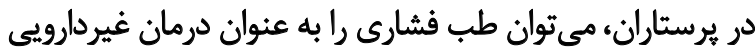

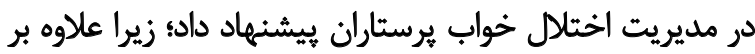

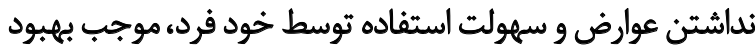
كيفيت خواب و درنتيجه كيفيت زندكى يرستاران مي تىشود. از نقاط قوت اين يروهش، ميتوان به مقايسه كيفيت خواب

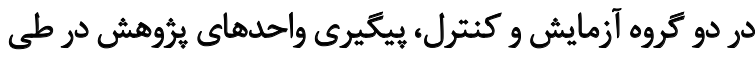

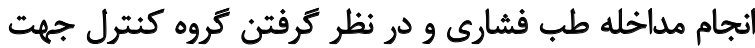

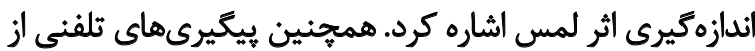

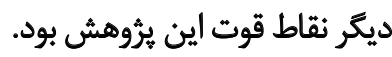

از جمله محدوديتهاى ثروهش حاضر مي توان به تفاوت

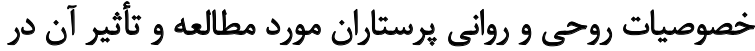

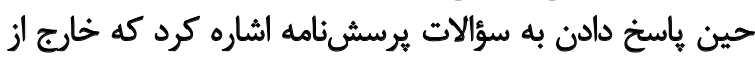

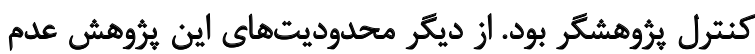

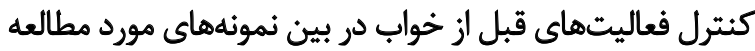

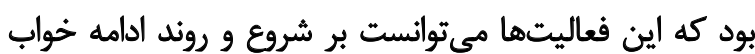

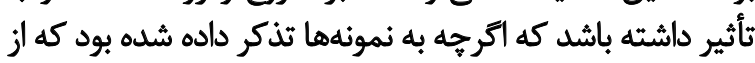

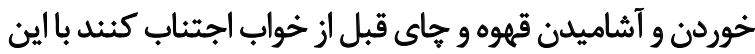

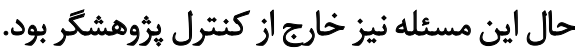

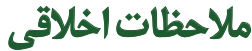

\section{يبروى أز اصول اخلاق بثوهش}

تمامى اصول اخلاق در يروهش در اين مقاله رعايت شده و

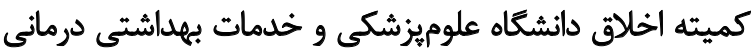

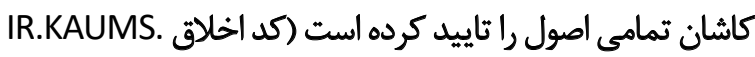

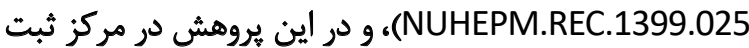
كارآزماييىهاي بالينى نيز ثبت شده است.

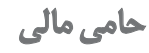

كعاونت يزوهشى دانشعاه علوميزشكى و خدمات بهداشتى برونى كاشان حامى مالى اين يُروهش بوده است.

$$
\text { مشار كت نويسند مكان }
$$

تدوين اوليه طرح: مهديه صابرى؛ ويرايش نهايى مقاله و آناليز آمارى: حسين اكبرى؛ جمع آورى دادهها: سجاد عباسى طادئ طادى. 


\section{References}

[1] Ayik C, Özden D. The effects of preoperative aromatherapy massage on anxiety and sleep quality of colorectal surgery patients: A randomized controlled study. Complementary Therapies in Medicine. 2018; 36:93-9. [DOI:10.1016/j.ctim.2017.12.002] [PMID]

[2] Weaver AL, Stutzman SE, Supnet C, Olson DM. Sleep quality, but not quantity, is associated with self-perceived minor error rates among emergency department nurses. International Emergency Nursing. 2016; 25:48-52. [DOI:10.1016/j.ienj.2015.08.003] [PMID]

[3] Dong H, Zhang Q, Sun Z, Sang F, Xu Y. Sleep problems among Chinese clinical nurses working in general hospitals. Occupational Medicine. 2017; 67(7):534-9. [DOI:10.1093/occmed/kqx124] [PMID]

[4] Griffiths P, Dall'Ora C, Simon M, Ball J, Lindqvist R, Rafferty AM, et al. Nurses' shift length and overtime working in 12 European countries: The association with perceived quality of care and patient safety. Medical Care. 2014; 52(11):975-81. [DOI:10.1097/ MLR.0000000000000233] [PMID] [PMCID]

[5] Johnson AL, Jung L, Brown KC, Weaver MT, Richards KC. Sleep deprivation and error in nurses who work the night shift. Journal of Nursing Administration (JONA). 2014; 44(1):17-22. [DOI:10.1097/ NNA.0000000000000016] [PMID]

[6] Bozorg Sohrabi F, Soleimani A, Habibi M, Emami Zaidi A. [Sleep quality of nurses working in the intensive care units of open heart surgery in hospitals of Mazandaran Province and related factors: A cross-sectional study (Persian)]. Nasim-e-Tandaresti Quarterly. 2014; 2(1):18-23. http://jfh.iausari.ac.ir/article_639702.html

[7] bahri N, Shamshri M, Moshki M, Mogharab M. [The survey of sleep quality and its relationship to mental health of hospital nurses (Persian)]. Iran Occupational Health. 2014; 11(3):96-104. http://ioh.iums. ac.ir/browse.php?a_id=972\&sid=1\&slc_lang=fa

[8] Çelik S, Taşdemir N, Kurt A, İlgezdi E, Kubalas Ö. Fatigue in intensive care nurses and related factors. The International Journal of Occupational and Environmental Medicine. 2017; 8(4):199-206. [DOI:10.15171/ijoem.2017.1137] [PMID] [PMCID]

[9] Korompeli A, Chara T, Chrysoula L, Sourtzi P. Sleep disturbance in nursing personnel working shifts. Nursing Forum. 2013; 48(1):45-53. [DOI:10.1111/nuf.12005] [PMID]

[10] Carney ML. The hidden shift: How do night shift nurses learn to adapt to circadian disruption. Journal for Nurses in Professional Development. 2013; 29(6):316-20. [DOI:10.1097/NND.0000000000000016] [PMID]

[11] Ozgoli G, Armand M, Heshmat R, Alavi Majd H. Acupressure effect on sleep quality in menopausal women (Persian)]. Commonwealth Magistrates \& Judges Association. 2012; 2(3):212-24. http://cmja. arakmu.ac.ir/browse.php?a_code=A-10-140-1\&sid=1\&slc_lang=fa

[12] Arami S, Kazemi M, Esmaeili-Nadimi A. [Comparing the effect of acupressure points shenmen (HE7) with a third eye on anxiety in patients undergoing coronary angiography (Persian)]. Medical-Surgical Nursing Journal. 2015; 4(2):41-6. https://www.sid.ir/en/Journal/ViewPaper.aspx?ID=456653

[13] Akbarzade M, Ghaemmaghami M, Yazdanpanahi Z, Zare N, Mohagheghzadeh A, Azizi A. Comparison of the effect of dry cupping therapy and acupressure at $B L 23$ point on intensity of postpartum perineal pain based on the short form of McGill Pain Questionnaire. Journal of Reproduction \& Infertility. 2016; 17(1):39-46. [PMID] [PMCID]

[14] Yeh ML, Chang YC, Huang YY, Lee TY. Complementary therapies in medicine: A randomized controlled trial of auricular acupressure in heart rate variability and quality of life for hypertension. Complementary Therapies in Medicine. 2015; 23(2):200-9. [DOI:10.1016/j. ctim.2015.01.005] [PMID]

[15] Liu YQ, Ren YL, Zhao L, Yu L, Chen J, \& Yang M, et al. [Analysis on characteristics of meridians and acupoints about acupuncture in treatment of insomnia based on data mining (Chinese)]. China Journal of Traditional Chinese Medicine and Pharmacy. 2015; 30:2734-7. [PMID]

[16] Su ZW, Ren YL, Zhou SY, Qin HZ, Chen DS, Liu T, Li Y. [Analysis on characteristics of meridians and acupoints of acupuncture and moxibustion for diarrhea in ancient based on data mining (Chinese)]. Zhongguo Zhen Jiu. 2013; 33(10):905-9. [PMID]

[17] Chen L, Sun LL, Wang X, Zhang F. Literature study on acupoint selection and prescription of acupuncture for treatment of insomnia. Journal of Traditional Chinese Medicine. 2012; 53(12):1051-4 https://en.cnki.com.cn/Article_en/CJFDTotal-ZZYZ201212024.htm.

[18] Wang Z, Hu X, Su J, Gao X, Xu N, \& Xing Y, et al. The efficacy and safety stimulating a single acu-point shenmen (HT 7) for managing insomnia: A systematic review of randomized controlled trials. European Journal of Integrative Medicine. 2017; 15:17-22. [DOI:10.1016/j. eujim.2017.08.010]

[19] Wu P, Cheng C, Song X, Yang L, Deng D, Du Z, et al. Acupoint combination effect of Shenmen (HT 7) and Sanyinjiao (SP 6) in treating insomnia: Study protocol for a randomized controlled trial. Trials. 2020; 21(1):1-9. [DOI:10.1186/s13063-020-4170-1] [PMID] [PMCID]

[20] Shariati A, Jahani S, Hooshmanda M, Khalili N. The effect of acupressure on sleep quality in hemodialysis patients. Complementary Therapies in Medicine. 2013; 20(6):417-23. [DOI:10.1016/j. ctim.2012.08.001] [PMID]

[21] Cerrone R, Giani L, Galbiati B, Messina G, Casiraghi M, Proserpio E ea. Efficacy of $\mathrm{HT} 7$ point acupressure stimulation in the treatment of insomnia in cancer patients and in patients suffering from disorders other than cancer. Minerva Medica. 2008; 99(6):535-7. [PMID]

[22] Kaur A, Kumar CN. Effectiveness of warm water foot bath on quality of sleep among hospitalized patients. International Journal of Health Sciences \& Research. 2017; 7(10):172-5. https://www.ijhsr. org/IJHSR_Vol.7_Issue.10_Oct2017/25.pdf

[23] Ahangar Anzabi A, Zare BahramAbadi M, Shafia Abadi A. [The effectiveness of occupational development based on super's growth theory on reducing students work-family conflicts and job burnout (Persian)]. Journal of Instruction and Evaluation. 2016; 9(33):45-67. https://www.sid.ir/en/Journal/ViewPaper.aspx?ID=518306

[24] Safa A, AdibHajbaghery M, FazelDarbandi A. [The relationship between sleep quality and quality of life in older adults (Persian)] Iranian Journal of Psychiatric Nursing. 2015; 3(3):53-62. http://ijpn.ir/ browse.php?a_code=A-10-1-69\&sid=1\&slc_lang=fa

[25] Buysse DJ, Reynolds lii CF, Monk TH, Berman SR, Kupfer DJ. The pittsburgh sleepuality index: A new instrument for psychiatric practice and research. Psychiatry Research. 1989; 28(2):193-213. [DOI:10.1016/0165-1781(89)90047-4] [PMID]

[26] Chan MF, Chan EA, Mok E. Effects of music on depression and sleep quality in elderly people: A randomised controlled trial. Complementary Therapies in Medicine. 2010; 18(3-4):150-9. [DOI:10.1016/j. ctim.2010.02.004] [PMID]

[27] Saeedi M, Ashktorab T, Shamsi khani S, Saatchi KT. The effect of progressive muscle relaxation on insomnia severity of hemodialysis patients. Complementary Medicine Journal of Faculty of Nursing \& 
Midwifery (CMJA). 2012; 2(2):34-46. https://www.sid.ir/en/journal/ ViewPaper.aspx id $=334842$

[28] Oujing LI, Wang F. [Acupuncture at back-points of five, Geshu (BL 17) and Shenmen (HT 7) for the treatment of menopausal insomnia (Chinese)]. Zhongguo Zhen Jiu = Chinese Acupuncture \& Moxibustion. 2018; 38(5):4693-72. [DOI:10.13703/j.0255-2930.2018.05.005] [PMID]

[29] Sun JL, Sung MS, Huang MY, Cheng GC, Lin CC. Effectiveness of acupressure for residents of long-term care facilities with insomnia: A randomized controlled trial. International Journal of Nursing Studies. 2010; 47(7):798-805. [DOI:10.1016/j.jinurstu.2009.12.003] [PMID]

[30] Simoncini M, Gatti A, Quirico PE, Balla S, Capellero B, Obialero R, et al. Acupressure in insomnia and other sleep disorders in elderly institutionalized patients suffering from Alzheimer's disease. Aging Clinical and Experimental Research. 2015; 27(1):37-42. [DOI:10.1007/ s40520-014-0244-9] [PMID]

[31] Yuan J, Wang H, Chen J, Lei Y, Wan Z, Zhao Y, et al. Effect of low frequency repetitive magnetic stimulation at Shenmen (HT7) on sleep quality in patients with chronic insomnia. Medicine. 2020; 99(30):e21292. [DOI:10.1097/MD.0000000000021292] [PMID] [PMCID]

[32] Backer M, Michael G. Acupuncture in the treatment of pain. An Integrative Approach United Kingdom: Churchill Livingstone; 2010.

[33] Saper C, Scammell TE, Lu J. Hypothalamic regulation of sleep and circadian rhythms. Nature. 2005; 437(7063):1257-63. [DOI:10.1038/ nature04284] [PMID]

[34] Shahdadi H, Allah-yari J, Mansouri A. [Effect of acupressure on sleep quality in patients with diabetes mellitus (Persian)]. Journal of Diabetes Nursing. 2017; 5(2):78-85. https://www.sid.ir/fa/journal/ ViewPaper.aspx?id=295880

[35] Jianguo Z. A newly compiled practical English-Chinese library of traditional chinese medicine: Gynecology of traditional Chinese medicine. Shanghai: Shanghai University of Chinese Medicine Press; 2007. https://www.purpleculture.net/a-newly-compiled-practicalenglishchinese-library-of-traditional-chinese-medicine-gynecology-oftraditional-chinese-medicine-p-14124/ 
This Page Intentionally Left Blank 